\title{
The Unique Observing Capabilities of the Swift X-ray Telescope
}

\author{
J.E. Hill $^{\mathrm{a}, \mathrm{b} 1}$, L. Angelinia ${ }^{\mathrm{a}, \mathrm{d}}$, D.C. Morris ${ }^{\mathrm{c}}$, D.N. Burrows ${ }^{\mathrm{c}}$, A.F. Abbey ${ }^{\mathrm{e}}$, S. Campana ${ }^{\mathrm{f}}$, \\ M. Capalbi ${ }^{\text {i }}$, G. Cusumano ${ }^{\text {, }}$, J.A. Kennea ${ }^{\mathrm{c}}$, R. Klar ${ }^{\mathrm{h}}$, C. Mangels ${ }^{\mathrm{h}}$, A. Moretti ${ }^{\mathrm{f}}$, \\ J.P. Osborne ${ }^{\mathrm{e}}$, M. Perri ${ }^{\mathrm{i}}$, J. Racusin ${ }^{\mathrm{c}}$, G. Tagliaferrif ${ }^{\text {', F. Tamburelli }}{ }^{\mathrm{i}}$, P. Wood ${ }^{\mathrm{h}}$, \\ J.A. Nousek ${ }^{\mathrm{c}}$, A. Wells ${ }^{\mathrm{c}, \mathrm{e}}$ \\ ${ }^{a}$ NASA/Goddard Space Flight Center, Greenbelt, MD 20771, USA, \\ ${ }^{\mathrm{b}}$ Universities Space Research Association, 10211 Wincopin Circle, Suite 500, Columbia, MD 21044, USA, \\ ${ }^{c}$ Department of Astronomy \& Astrophysics, Penn State University, USA, \\ ${ }^{\mathrm{d}}$ Department of Physics \& Astronomy, The Johns Hopkins University, Baltimore, MD 21218, USA, \\ ${ }^{\mathrm{e}}$ Physics \& Astronomy Department, University of Leicester, LE1 7RH, UK, \\ ${ }^{\mathrm{f}}$ INAF Osservatorio Astronomico di Brera, Milano, Italy, \\ ${ }^{g} \mathrm{CNR}$ Istituto di Fisica Cosmica ed Applicazioni dell Informatica, Italy, \\ ${ }^{\mathrm{h}}$ Southwest Research Institute, San Antonio, TX, USA \\ ${ }^{\mathrm{i}}$ ASI-ASDC, Via G. Galilei, I-00044 Frascati, Italy
}

\begin{abstract}
The XRT is a sensitive, autonomous X-ray imaging spectrometer onboard the Swift Gamma-Ray Burst Observatory. The unique observing capabilities of the XRT allow it to autonomously refine the Swift BAT positions $\left(\sim 1-4^{\prime}\right.$ uncertainty) to better than 2.5 arcsec in XRT detector coordinates, within 5 seconds of target acquisition by the Swift Observatory for typical bursts, and to measure the flux, spectrum, and light curve of GRBs and afterglows over a wide dynamic range covering more than seven orders of magnitude in flux ( $62 \mathrm{Crab}$ to $<1 \mathrm{mCrab}$ ). The results of the rapid positioning capability of the XRT are presented here for both known sources and newly discovered GRBs, demonstrating the ability to automatically utilise one of two integration times according to the burst brightness, and to correct the position for alignment offsets caused by the fast pointing performance and variable thermal environment of the satellite as measured by the Telescope Alignment Monitor. The onboard results are compared to the positions obtained by ground-based follow-up.

After obtaining the position, the XRT switches between four CCD readout modes, automatically optimising the scientific return from the source depending on the flux of the GRB. Typical data products are presented here.
\end{abstract}

Keywords: CCDs, Gamma-Ray Bursts, X-rays, Swift, X-Ray Telescope, Centroiding, Readout Modes

\section{INTRODUCTION}

The Swift Gamma-ray Burst Explorer ${ }^{8}$ was launched on $20^{\text {th }}$ November 2004 to study gamma-ray bursts (GRBs) over a broad energy band covered by three instruments; a wide field of view gamma-ray Burst Alert Telescope (BAT) and two Narrow Field Instruments (NFIs); an X-ray Telescope (XRT) and a UV-Optical Telescope (UVOT). Swift slews rapidly to bursts detected by the Burst Alert Telescope (BAT) ${ }^{1}$, in order to point the $\mathrm{XRT}^{3}$ and $\mathrm{UVOT}^{17}$ telescopes at the burst for rapid follow-up observations of the afterglow.

The XRT is a focussed X-ray Telescope utilizing a grazing incidence Wolter 1 mirror module, a flight spare from the JET-X program, and an e2v CCD-22 at the focal plane. The XRT has five operating modes ${ }^{11}$; Image Mode which provides the initial centroiding capability when Swift first settles on a GRB, two Photodiode Modes which provide high timing resolution $(10 \mu$ second), Windowed Timing Mode for moderate timing (1.8 ms) and 1-dimensional position and Photon-Counting Mode for 2-dimensional position and maximum sensitivity. Each of the modes is optimized to provide the maximum science return according to the source count-rate.

During the observatory commissioning and calibration phase, which ended on the $5^{\text {th }}$ April 2005, the XRT modes were verified extensively. The ground operations ${ }^{13}$ and XRT mode parameters have been optimized to mitigate the effects of operating the $\mathrm{CCD}$ between $-70^{\circ} \mathrm{C}$ and $-45^{\circ} \mathrm{C}$ due to the XRT cooling sub-system, which was expected to cool the CCD

\footnotetext{
${ }^{1}$ Correspondence: Email: jhill@milkyway.gsfc.nasa.gov: Phone: +1 301 286-0572; Fax: +1 301 286-1684; Building 2, Code 662, NASA/Goddard Space Flight Center, Greenbelt, MD 20771, USA
}

UV, X-Ray, and Gamma-Ray Space Instrumentation for Astronomy XIV edited by Oswald H. W. Siegmund, Proc. of SPIE Vol. 5898 589815, (2005) - 0277-786X/05/\$15 - doi: 10.1117/12.618026 
to $-100{ }^{\circ} \mathrm{C}$ and to control the temperature to $+/-1{ }^{\circ} \mathrm{C}$, failing to operate 10 days after launch. Furthermore, the XRT onboard software was updated on $26^{\text {th }}$ May 2005 to improve the operating range over which the flight software could account for the increasing dark current and hot pixels. Reflections of the bright Earth into the telescope when the lineof-sight approaches the Earths limb also increased the complexity of optimizing the modes for on-orbit operations. Nevertheless, the XRT observed its first GRB on $23^{\text {rd }}$ December $2004^{4}$, and the first automated response to a burst occurred on $17^{\text {th }}$ January 2005 , when the XRT was successful in obtaining a centroid position of the burst while the gamma-ray phase was still in progress ${ }^{12}$. To date $\left(21^{\text {st }}\right.$ July 2005) the XRT has detected 42 of the 46 BAT triggered bursts; the remaining four were constrained by the sun and were not followed up by Swift. Two bursts were initially constrained by the moon, but successfully followed up and detected by the XRT several days later. The spacecraft slewed promptly (in less than 4 minutes) to 24 of the bursts; the remaining 16 were delayed due to the Earth limb obscuring the target or due to the observatory being in an engineering mode. A successful onboard centroid was obtained for 11 bursts, 9 were too faint to centroid and one gave a false centroid on a cosmic-ray (prior to the software upgrade). Three of the prompt follow-up observations were while XRT was performing calibration observations in Manual State.

Cross-calibration has been performed with XMM-Newton ${ }^{17}$ and $\mathrm{RXTE}^{7}$ for spectral and temporal comparisons, respectively. The XRT fluxes and timing have been found to correlate well with the results from the other missions. Similarly, fluxes and timing have been calibrated between the $\mathrm{XRT}^{12}$ and the $\mathrm{BAT}^{7}$ and were also found to correlate well.

The XRT modes were described in detail previously ${ }^{11}$ with reference to the ground calibration results. An overview of the modes will be provided here, including updates, which have been made to the onboard software. We present the onorbit performance of the XRT modes, focusing on the centroiding capability in particular.

\section{DESCRIPTION OF THE XRT DATA MODES}

\subsection{Overview}

The nominal operating state for the XRT is Auto State, where the onboard software determines which mode the XRT should be in according to whether the source is a new GRB (Automated Target or AT) or a Pre-Planned Target (PPT) and according to the count rate as measured by the onboard software. A target is designated as an AT when the BAT triggers on an un-catalogued source due to a change in count rate localized to a point on the sky. In addition, a Target of Opportunity (TOO) maybe uploaded to the observatory via telecommand from the ground with the 'is_new_at' flag set to true. A PPT may be either a target scheduled in the Pre-Planned Science Timeline (PPST) or an automated observation of a new GRB, but after the first orbit. If the target is designated an AT, when the spacecraft settles on the target, the XRT will switch from Low-rate Photodiode (LrPD) Mode into Image Mode and attempt to centroid on the source. Following Image Mode, the XRT will switch into Piled-up Photodiode (PuPD) Mode, LrPD, Windowed Timing (WT) Mode and Photon-Counting (PC) Mode according to the count-rate. It is also possible for the XRT to switch back up through the modes if the count-rate should increase. If the target is a PPT, the XRT continues in LrPD Mode after the spacecraft settles and then switches to the appropriate mode according to the count-rate. The spacecraft will not allow the narrow field instruments to point within 28 degrees from the Earth limb, 14 degrees from the Moon or 45 degrees from the Sun (these are the current settings and may be optimized further). This means that, as Swift continues around its orbit, each target will eventually become obscured by the Earth's limb and the spacecraft will slew to the next scheduled target. As soon as the spacecraft begins to slew the XRT obtains a Bias Row, a Bias Map and then switches into LrPD Mode for the remainder of the slew until the spacecraft settles. A flow diagram of the XRT observing sequence is shown in Figure 2.1.

During most orbits, Swift travels through the South Atlantic Anomaly (SAA). The large number of proton events would flood the XRT telemetry and therefore the XRT onboard software monitors the spacecraft longitude and latitude and, when the current position lies within one of the four circles uploaded to the flight software (FSW) to define the SAA, the XRT switches into Null Mode, where the software continues to clock out the CCD to prevent the build up of charge, but it does not telemeter any events. The onboard software can also be configured with the SAA tracking disabled or set to the Attitude Control System (ACS) 'in_SAA' flag which is sent from the spacecraft to the instruments as a telecommand across the MIL-STD-1553B bus along with the current longitude, latitude, 'is_settled' flag, 'in_10_arcmin' flag, etc. If a burst should occur while Swift is in the SAA, the software will switch from Null Mode 
into Image Mode to attempt to obtain a position. The software will also obtain a partial PuPD frame before switching back into Null Mode for the remainder of the SAA. When the onboard software determines that Swift has exited the SAA (as defined by the four circles), the XRT switches back into LrPD and into the appropriate mode according to the count-rate.

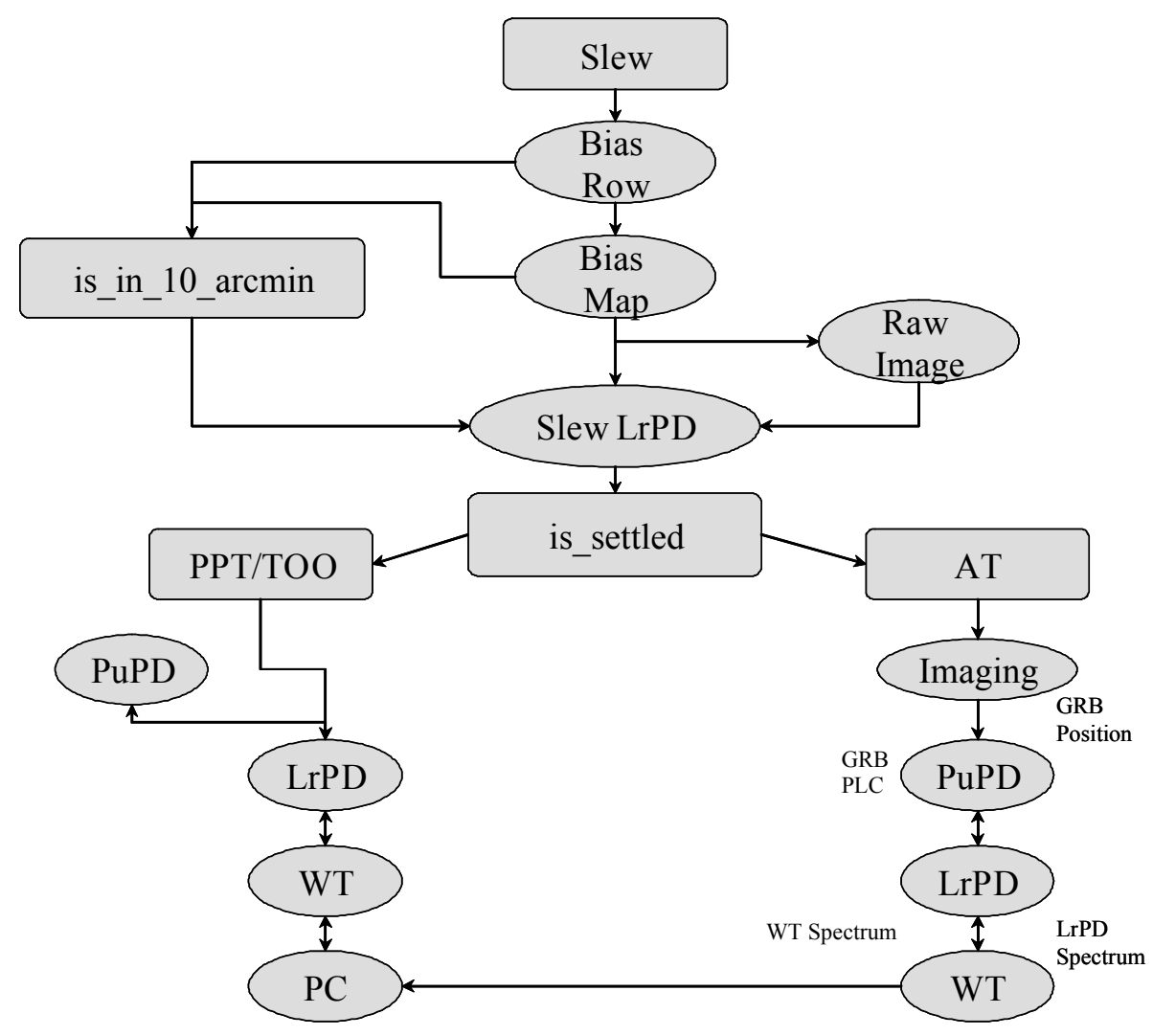

Figure 2.1 The Automated operation of XRT. The switch points for the different modes are as follows; PuPD to LrPD $=20000$ cps; LrPD to PuPD = 25000 cps; LrPD to WT $=200 \mathrm{cps} ; \mathrm{WT}$ to $\mathrm{LrPD}=600 \mathrm{cps} ; \mathrm{WT}$ to $P C=2 \mathrm{cps} ; \mathrm{PC}$ to $\mathrm{WT}=10 \mathrm{cps}$

\subsection{Image Mode}

A primary function of the XRT is to provide a position update to the BAT calculated position, accurate to 5 arcsec. The BAT determines a GRB position accurate to approximately 4 arcmin, and the spacecraft slews to put the BAT position in the centre of the field of view (FOV) of the Narrow Field Instruments (NFI's). If the source is bright enough, the XRT refines this position and telemeters it through the Tracking and Data Satellite System (TDRSS) within seconds of the spacecraft settling on the target. This position is sent out through the Gamma-ray Co-ordinate Network (GCN) to allow ground-based telescopes to perform follow-up observations. In addition, the position is sent as an instrument-toinstrument telecommand to allow the UVOT to optimize its processing on a smaller region centred on the XRT position and to the Figure of Merit processor, which schedules the observations, to request follow-up observations using the more accurate position.

The XRT has two integration times in Image Mode; a short 0.1 second exposure and, if the source is not bright enough (i.e. less than 20 counts in the centroiding window), it processes a second 2.5 second integration image which has accumulated while the previous frame was being readout. According to this process, the position can take up to 5.2 seconds to be telemetered. If the centroid sigma is too large or the position does not converge (both are uploadable parameters), the software will restart the clocking sequence starting with a 0.1 second integration. When Swift was launched, the XRT software was configured to attempt to centroid up to twelve times before sending a Centroid Error Message with a flag indicating the type of error; whether the source was not bright enough for an accurate centroid, 
whether the sigma was too large and therefore a cosmic-ray was contaminating the field or, if the centroid position did not converge, in which case another bright source was in the field.

On-orbit it has been found that the rate of decay in flux ${ }^{9,12,24}$ between the burst and the afterglow is much faster than initially anticipated and therefore, in a number of cases where the burst was obscured by the Earth limb and the slew was delayed more than a few minutes, the burst is fainter than the limit for a centroid when Swift finally observes with the NFI's. From on-orbit experience we have found that if there are no source counts and if there is a Cosmic-ray event in the corner of the CCD near the events from the calibration sources, the software is unable to detect that this is not a real source and therefore may telemeter an incorrect centroid position. To restrict this behaviour the corner sources were initially eliminated from the field with the use of the bad pixel map (this also restricts the field of view in PC Mode), and more recently with the Build 8.9 flight software the corner sources are eliminated from the processing field only in Image Mode. Furthermore, the number of attempts to centroid has been reduced from twelve to three as from the onorbit data a large number of centroid attempts increased the chance of a cosmic-ray interaction near the corner sources or near a background X-ray event considerably, and the chance of a GRB re-brightening enough to centroid later was very unlikely given the rapid decay seen so far.

Several parameters have been optimized following the on orbit calibration effort:

$\circ$ Centroiding window size: 25 x 25 , half size.

- Sigma_max: 12, defining the PSF for bright sources.

- Iterations: $<4$, usually converges within 3 iterations.

○ Minimum number of counts to centroid: 20 defines the minimum source strength to centroid, $\sim 5.6$ mCrab.

Image Mode is operated in low gain. Due to the expectation that the X-rays will be piled-up, i.e. more than one X-ray will have interacted in a single pixel or in adjacent pixels, only limited spectroscopy can be obtained from the wings of the Point Spread Function (PSF) and therefore we obtain a higher maximum flux by optimizing the maximum Analogue-to-Digital Conversion (ADC) channel to be the full-well of the CCD pixels ( $~ 80000$ electrons) and under sample the spectrum. For the other modes, all of which are operated in high gain, the maximum ADC value corresponds to the highest X-ray energy that it is possible for the XRT to detect, i.e. $\sim 10 \mathrm{keV}$. The ADC to energy conversion for low and high gain modes are $80 \mathrm{eV} / \mathrm{dn}$ and $2.5 \mathrm{eV} / \mathrm{dn}$, respectively (the conversion is affected by temperature, primarily effecting the high gain operation ${ }^{14}$ ).

\subsection{Piled-up and Low-rate Photodiode Mode}

Photodiode mode is the highest timing resolution mode (10 $\mu$ second), designed for the very highest fluxes $(200 \mathrm{mCrab}$ - $62 \mathrm{Crab}$ ), when rapid changes in the light-curve are expected. The readout scheme clocks a row into the readout register and then reads out a pixel; then the next row is clocked into the readout register and so on. Essentially, all the charge in the CCD image is binned into a single pixel at the output, but the exposure time of each of the pixels summed together is not from the same instance. There is no imaging capability in this mode, but high-resolution spectroscopy can be achieved for fluxes below 4.6 Crab.

During Observatory level thermal vacuum tests, it was found that temperature variations in the XRT electronics and in the Focal Plane Camera (FPCA) caused the conversion of mean noise value into digital number (DN) to change. For Windowed Timing and Photon Counting Mode this was accounted for by the fact that the temperature changes are slow and the Bias Row and Bias Map are updated every time the spacecraft slews; therefore, as long as the changes were small and the noise peak value did not increase above the event threshold, this was taken into account. In Photodiode Mode the increase in the mean DN value of the noise was exacerbated by the fact that the clocking scheme sums the pixels from the entire array and only an event threshold was applied to the data. Therefore, as the value of the ADC channel of the mean noise increased, the noise events exceeded the event threshold and were interpreted by the flight software as X-ray events. This meant that the count-rate evaluated onboard was dominated by the noise events and XRT was switching into the incorrect mode. Software was implemented prior to launch for Low-rate Photodiode Mode to calculate the bias level from the mean value of all the pixels in a frame below a bias threshold and to subtract that bias from the next frame. When the instrument switches into Piled-up Photodiode mode the last bias value calculated in LrPD Mode is subtracted from the PuPD Mode data. 


\subsection{Windowed Timing Mode}

Windowed Timing Mode has 1-dimensional imaging capability, $1.8 \mathrm{~ms}^{2}$ timing resolution and provides high-resolution spectroscopy for fluxes between 1-600 milli-Crab. The timing resolution is achieved by summing 10 rows in the readout register $(15 \mu \mathrm{sec} / \mathrm{row})^{2}$ and only performing analogue to digital conversion for the central 200 pixels at the standard XRT rate of $6.6 \mu \mathrm{secs} /$ pixel; the remaining 400 pixels are fast clocked with no conversion.

A Bias Row is calculated every slew; however, if the Bias Row is aborted due to the spacecraft pointing within 10 arcmin of the target, the last Bias Row is retained and used. The Bias Row is subtracted from every row of Windowed Timing data and only the pixels above the event threshold are telemetered to the ground. Post-processing event reconstruction is performed on the ground by XRTDAS. Windowed Timing Mode uses a Bad Pixel Row, which is used to identify pixels that should be ignored by the onboard flight software due to having values above the event threshold but arising from hot pixels.

\subsection{Photon-Counting Mode}

Photon-Counting (PC) Mode is the more traditional mode of operation for an X-ray CCD and is the most sensitive XRT mode measuring fluxes lower than $2 \times 10^{-14} \mathrm{ergs} \mathrm{cm}^{-2} \mathrm{~s}^{-1}$ in $10 \mathrm{ksec}$. PC Mode provides 2-dimensional imaging, high spectral resolution and low timing resolution ( 2.5 seconds) for fluxes less than 1 milli-Crab. Thirty-one overclocks (additional pixels clocked from the serial register at the end of a row which are not from the image array) are used in PC Mode and the Bias Map calculation to determine a baseline level that is subtracted from every pixel in the corresponding row. Following the baseline correction, the bias-level is subtracted on a pixel-by-pixel basis using the Bias Map, which is updated every slew. Event recognition is performed onboard for every pixel that is above the event threshold and a $3 \times 3$ array is telemetered for each, centred on the local maximum.

The Bias Map is updated every slew, providing that the spacecraft pointing is not within 10 arcmin of the target before the algorithm is complete. Pixels identified as 'hot pixels' can be uploaded to the onboard Bad Pixel Map so that they are eliminated from the count-rate evaluation and from the event recognition. The baseline correction, bad pixel map and bias subtraction can be disabled in the onboard processing.

\subsection{TDRSS Products}

In addition to the XRT position and Image or the XRT Centroid Error from Image Mode, the XRT also telemeters two other quick-look products though TDRSS; a Prompt Light-Curve (PLC) and two TDRSS spectra. The PLC is the onboard count-rate, mode and frame duration for each of the first 100 frames of data following Image Mode. Two TDRSS spectra are generated; the first with LrPD Mode data, which is telemetered when the XRT switches from LrPD to WT Mode and a WT Mode spectrum which is telemetered when XRT switches into PC Mode. The LrPD Mode spectrum includes the lines from the ${ }^{55} \mathrm{Fe}$ calibration sources. No event recognition is performed to generate the TDRSS spectrum or light-curve.

\subsection{Telescope Alignment Correction}

To provide the required pointing accuracy and knowledge necessary for the XRT to obtain GRB positions accurate to 5 arcsecs, the spacecraft Star Trackers are mounted on the XRT forward telescope tube. In addition, the thermal variations seen by the telescope due to the Low Earth Orbit are controlled by eight bands of heaters mounted around the circumference of the forward and aft telescope tubes. Nevertheless, some bending of the tubes is expected due to changing thermal gradients as Swift moves around its orbit. The Telescope Alignment Monitor (TAM) Assembly (Figure 2.2) provides a measure of the movement of the FPCA and Star Trackers relative to the mirror optical axis via an LED mounted in the FPCA. The TAM LED shines up the aft tube through a light baffle to a beam-splitter mounted on the Optical Bench Interface Flange (OBIF). The beam is split such that half the light from the LED is reflected onto the TAM camera, mounted opposite the beam-splitter on the OBIF, creating a 'primary' spot; the other half travels up through the forward tube and is reflected off a mirror flat mounted on the Star-Tracker radiator plate back towards the beam-splitter. The secondary beam makes a second reflection at the beam-splitter and illuminates the TAM camera with a secondary spot. The positions of these two spots are measured by the TAM software via a centroid calculation within a window around each of the spots. The GRB position determination software (Imaging Mode, see section 2.2)

\footnotetext{
${ }^{2}$ This is an update to Hill et al. 2004.
} 
compares the 'new' positions of the two spots with the default positions. The default positions were measured during the initial on-orbit alignment when the telescope was in a uniform thermal environment. This enables a GRB position to be corrected for any thermal variations affecting the pointing of the Star-Trackers, and therefore the absolute pointing direction on the sky, and the variations affecting the focus position of the GRB onto the CCD. The deviations of the two spots from the default positions are folded into the GRB position calculation in Image Mode.

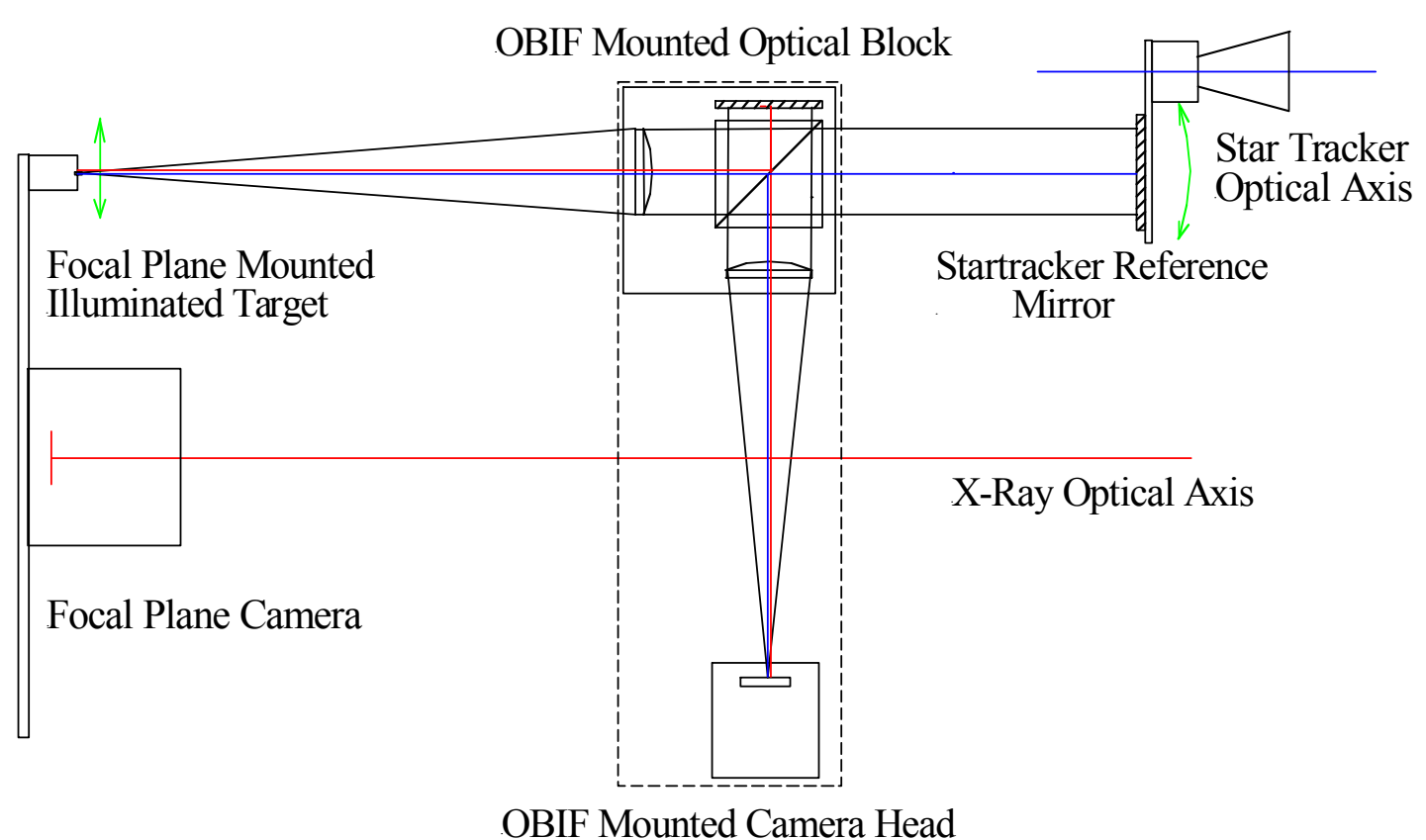

Figure 2.2 Telescope Alignment Monitor (TAM) and Assembly

\subsection{On-orbit updates to the XRT software}

\subsubsection{Temperature Dependent Threshold Table for all Modes}

Due to the loss of CCD temperature control, in some circumstances when the $\mathrm{CCD}$ temperature rises above $-48^{\circ} \mathrm{C}$ the dark current becomes greater than the bias threshold, insufficient bias is subtracted and therefore the dark current eventually becomes greater than the event threshold. The flight software interprets this as an increase in source countrate and switches into a higher event mode, eventually switching into Photodiode Mode. Because of the additional parallel transfers in Photodiode mode, the clocking sequence requires more power than the other modes, such that it actually begins to heat up the CCD. Without temperature control, the system can end up in a run-a-way condition due to the count-rate rising with temperature and Photodiode Mode increasing the temperature. Unless the spacecraft slews to a new target (putting the XRT radiator in a colder orientation ${ }^{13}$ ) the system maintains this condition and the telemetry is flooded with noise events, eventually overflowing the XRT telemetry buffer and filling up the XRT data partition on the spacecraft. To mitigate this effect, the onboard software was updated on $26^{\text {th }}$ May 2005 to use a temperature dependent threshold table. This includes an event threshold and a bias threshold for each of the modes for a configurable number of temperature ranges. Currently the XRT has thresholds defined for temperatures below $-60{ }^{\circ} \mathrm{C}$ and for every degree between $-60{ }^{\circ} \mathrm{C}$ and $-40{ }^{\circ} \mathrm{C}$. For temperatures above $-40{ }^{\circ} \mathrm{C}$, where the data become severely degraded, the event threshold for all modes is set to 4095 (the maximum ADC channel, which has the effect of disabling the telemetry of any events. 


\subsubsection{Addition of Sigma Minimum Parameter in Imaging Mode}

From the on-orbit calibration of Image Mode we have determined that, in the case where there are very few true source counts, large cosmic-ray events which occur near isolated X-ray events from the background could be interpreted by the flight software as the source and could produce a false centroid position. From looking at the values of sigma telemetered in the Postage Stamp header one can see that, in this circumstance, a minimum sigma would be a better way to eliminate false centroids. This additional parameter was added to the flight software in May 2005.

N.B. On $27^{\text {th }}$ May 2005 the CCD sustained damage from a suspected micro-meteoroid impact. This has resulted in a very bright column, which at high temperatures floods adjacent columns down the centre of the CCD, and several other hot spots for which the number of pixels affected changes with temperature. For Windowed Timing Mode and for Photon-Counting and Image Mode the hot columns have been masked out in the onboard Bad Pixel Row and Bad Pixel Map, respectively. This alleviates the issue except at temperatures above $-48^{\circ} \mathrm{C}$. Currently, the Photodiode Modes have been de-activated until they have been re-calibrated. The hot columns have the effect of adding to the bias level in Photodiode Mode. As the temperature changes the amount of charge created by the hot pixels changes and therefore has the effect of changing the bias level with temperature. With the onboard temperature dependent threshold table, the bias threshold can be adjusted correctly with temperature so that the correct bias is calculated and subtracted. Assuming the affect of the hot pixels remain constant for a given temperature, the Photodiode Modes will be operational despite the damage, with some loss of the upper energy range.

\section{ON-ORBIT RESULTS}

\subsection{XRT Positions}

Calibration of the centroiding algorithm was performed with a variety of sources covering a large range of fluxes, 6.7 mCrab - 2.3 Crab. The positions according to SIMBAD ${ }^{3}$ in J2000 co-ordinates are shown in Table 3.1 and Figure 3.1 with the average position calculated by the onboard software for multiple observations. The onboard positions for each source were averaged over the total number obtained. It can be seen in Figure 3.1 that the deviations between the average flight software position and the SIMBAD J2000 positions are centred close to zero. The accuracy of the position is dependent on the number of counts from the source in the image and therefore, depending on whether the algorithm used a 0.1 second or 2.5 second integration, affects the absolute accuracy and creates a discontinuity in the accuracy versus source brightness. The flight software uses the longer integration time for the fainter sources, until the number of counts is sufficient to use the shorter integration, at which time the total number of counts from the short integration of the brighter source is less than that for the longer integration of the fainter source and therefore the accuracy is reduced. The effect can be seen in Figure 3.2, which shows the onboard centroiding accuracy versus approximate source intensity $(\sim 0.7$ counts $/ \mathrm{mCrab}$, with no pile-up correction) where the software is configured to require a minimum of 20 counts above threshold in the centroiding window before attempting to centroid.

Table 3.1 XRT onboard positions for calibration targets

\begin{tabular}{|l|r|r|r|r|r|}
\hline Source & RA (J2000) & DEC (J2000) & Roll (S/C Ave) & FSW RA & FSW Dec \\
\hline 4U 0614+091 & 94.2804 & 9.1369 & 255.9636 & 94.2808 & 9.1376 \\
\hline Cyg X-1 & 299.5903 & 35.2016 & 161.0563 & 299.5899 & 35.2008 \\
\hline Cyg X-2 & 326.1717 & 38.3217 & 192.0851 & 326.1712 & 38.3220 \\
\hline Cyg X-3 & 308.1074 & 40.9578 & 171.4018 & 308.1067 & 40.9584 \\
\hline EXO 0748-676 & 117.1388 & -67.7499 & 200.6094 & 117.1416 & -67.7515 \\
\hline Her X-1 & 254.4576 & 35.3424 & 120.9207 & 254.4571 & 35.3424 \\
\hline Sco X-1 & 244.9795 & -15.6402 & 102.1215 & 244.9791 & -15.6407 \\
\hline
\end{tabular}

\footnotetext{
${ }^{3}$ http://simbad.u-strasbg.fr/Simbad
} 


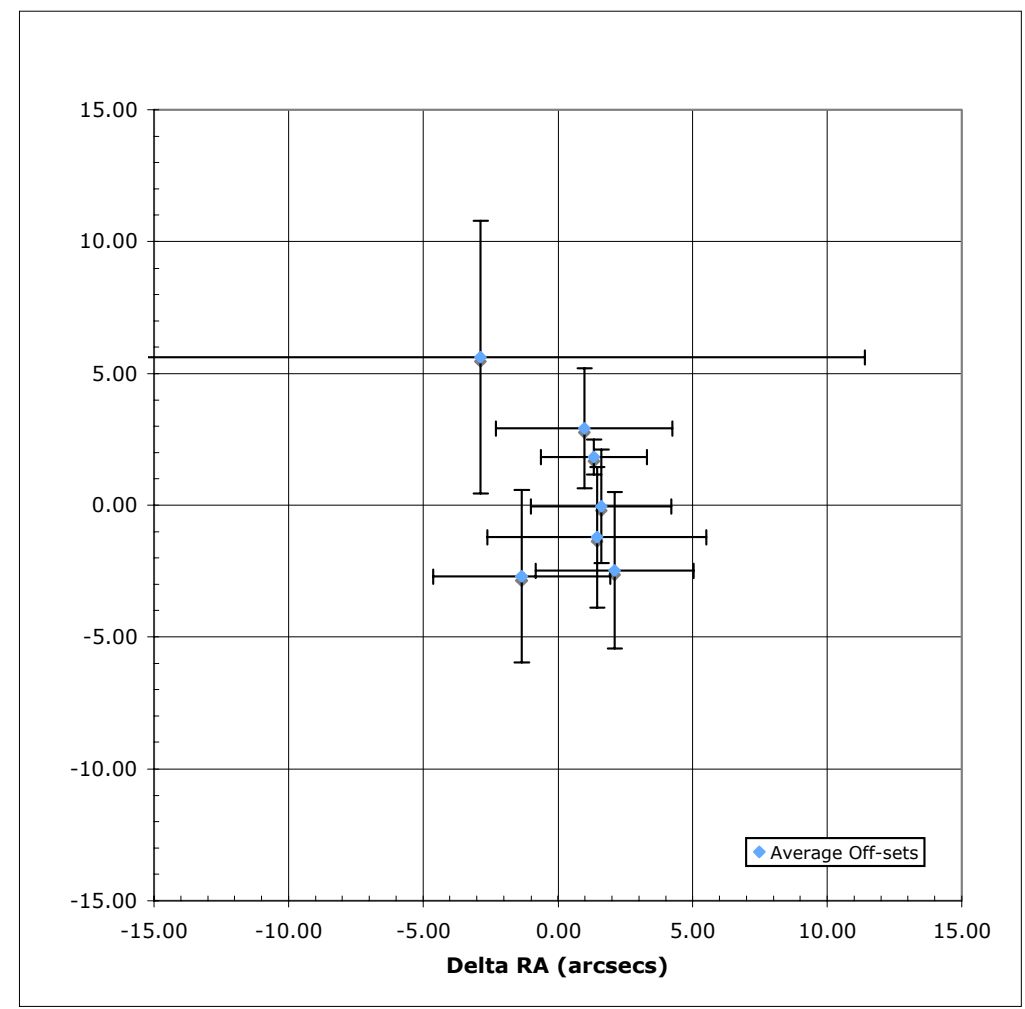

Figure 3.1 XRT centroiding accuracy for the calibration sources

If one takes all the positions of all the calibration sources and creates a histogram of the difference between the measured positions and the J2000 catalogued positions, one can see that there is an off-set in RA and Dec (Figure 3.3, left) of around 2 arcsecs. To verify the TAM correction, an idl script was written to reproduce the onboard FSW GRB position calculation using the values for the spacecraft position, TAM correction, and the source position in CCD coordinates from the TDRSS position and postage stamp messages. The calculation was verified against the positions obtained from the flight software and found to reproduce the flight values within the accuracy of the code $(\sim 0.1 \%)$. The TAM correction was then removed from the idl script and the positions for the calibration sources were re-calculated. A histogram of the positions was generated and is shown in Figure 3.3 (right). From comparing the two histograms, with and without the TAM correction, it can be seen that the TAM correction significantly improves the position accuracy as the histogram narrows. In addition, it can be seen that the TAM correction removes a significant offset in RA, shifting the mean from 4 arcsecs to 2 arcsecs. From these results the boresight offset, which causes the $\sim 2$ arcsec off-set in RA and DEC, will be uploaded to the flight software, improving the accuracy of the GRB position. 


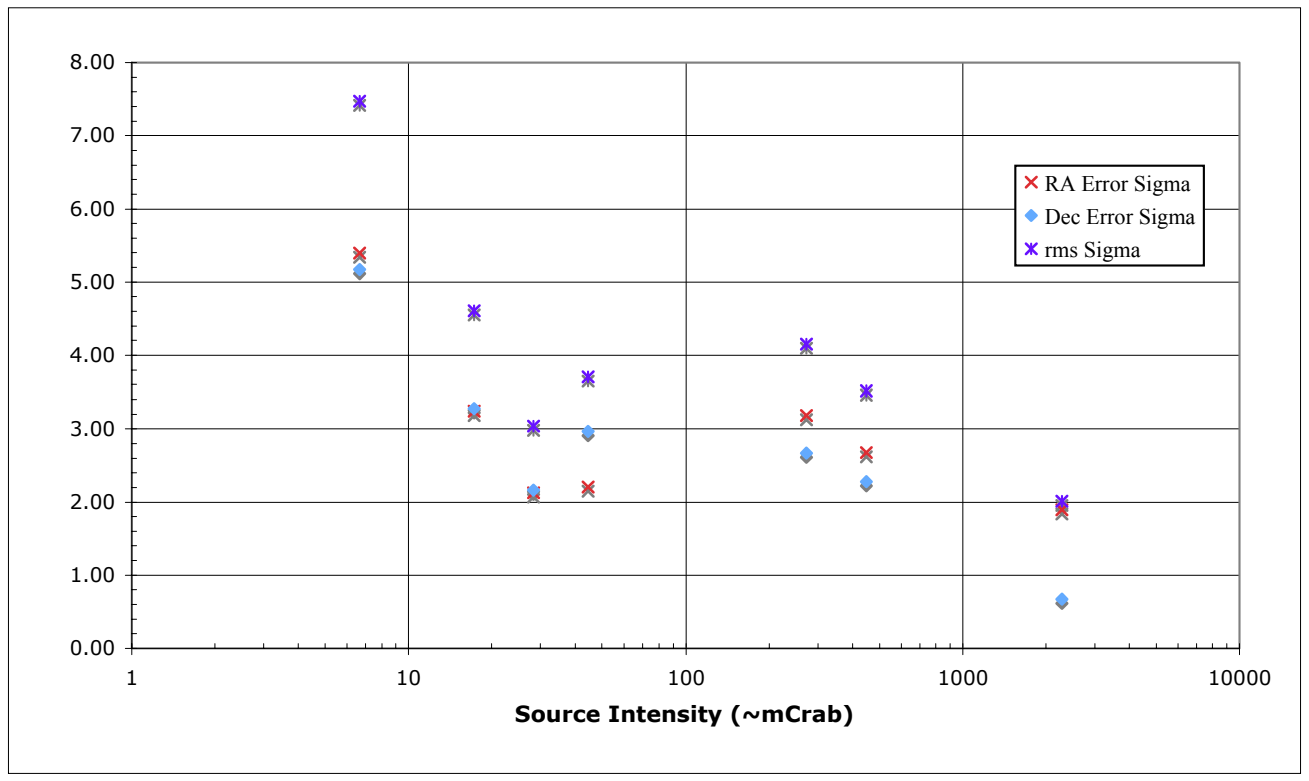

Figure 3.2 XRT centroiding accuracy versus source intensity (uncorrected for pile-up), where the XRT response is $\sim 0.7$ counts $/ \mathrm{mCrab}^{4}$
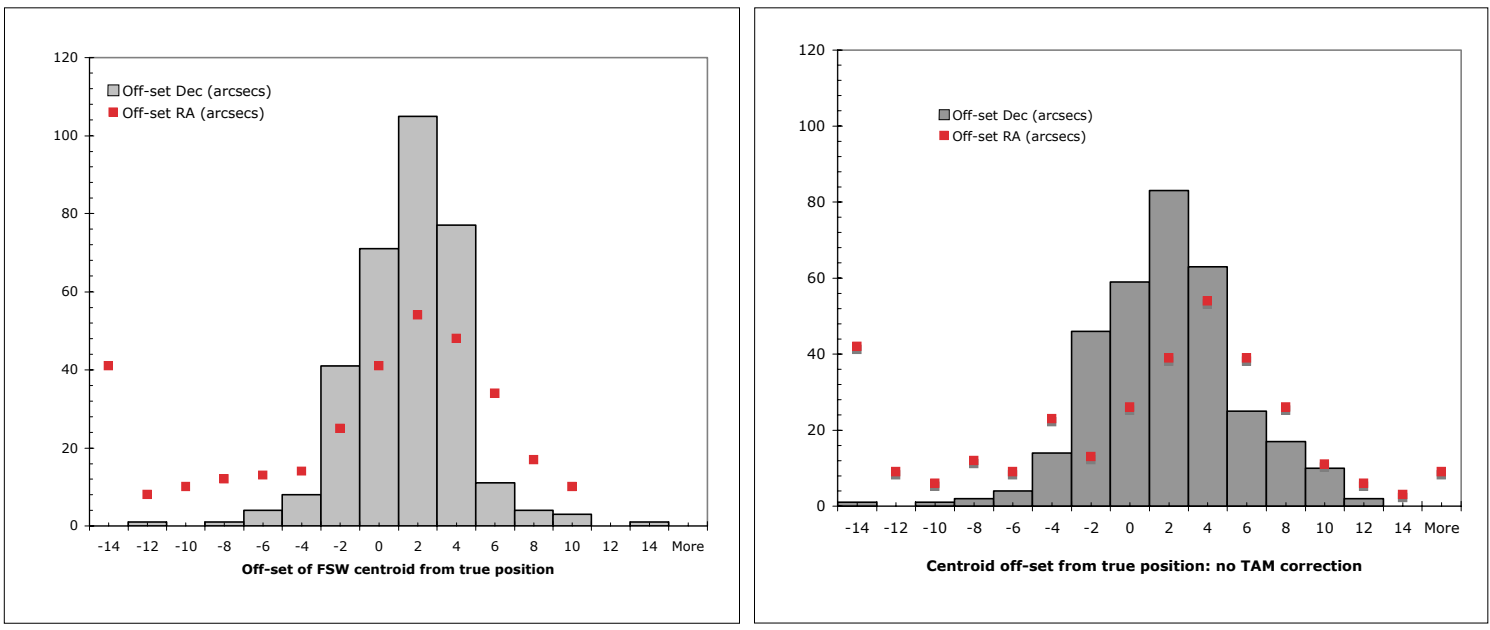

Figure 3.3 Histograms of the difference in RA and DEC between the J2000 positions and the FSW obtained centroids including the TAM correction (left) and the FSW centroids with no TAM correction (right)

\footnotetext{
${ }^{4}$ Note that the count-rate will be underestimated for the bright sources where the counts are piled up
} 
Table 3.2 XRT GRB positions from fast autonomous follow-up

\begin{tabular}{|c|c|c|c|c|c|c|c|c|c|c|c|c|c|c|}
\hline \multicolumn{3}{|c|}{ GRB/Trigger } & \multicolumn{12}{|c|}{ Observations } \\
\hline Trig & Date & $\begin{array}{l}\text { Time } \\
\text { UT }\end{array}$ & $\begin{array}{c}\text { BAT } \\
\text { RA }\end{array}$ & $\begin{array}{c}\text { BAT } \\
\text { Dec }\end{array}$ & \begin{tabular}{|c|} 
BAT \\
Error \\
\end{tabular} & \begin{tabular}{|c|} 
FSW \\
XRT RA
\end{tabular} & \begin{tabular}{|c|c} 
FSW \\
XRT Dec
\end{tabular} & $\begin{array}{c}\text { XRT } \\
\text { Error }\end{array}$ & $\begin{array}{l}\text { XRT RA } \\
\text { w/ TAM }\end{array}$ & \begin{tabular}{|c|} 
XRT DEC \\
w/ TAM
\end{tabular} & \begin{tabular}{|l|} 
XRT RA \\
no TAM \\
\end{tabular} & \begin{tabular}{|c|} 
XRT DEC \\
no TAM
\end{tabular} & Opt RA & Opt Dec \\
\hline 146970 & $5 / 7 / 21$ & $4: 29: 14$ & 253.445 & -28.39 & 4 & 253.4364 & -28.3815 & 5.8 & 253.4368 & -28.3818 & 253.4375 & -28.3805 & 253.4355 & $-28.3811^{6}$ \\
\hline 145994 & $5 / 7 / 14$ & $22: 40: 32$ & 169.694 & -15.528 & 4 & 169.6990 & -15.5479 & 5.8 & 169.6993 & -15.5480 & 169.7004 & -15.5473 & 169.6988 & $-15.5477^{5}$ \\
\hline 145754 & $5 / 7 / 13$ & 12:09:33 & 307.843 & 60.92 & 4 & 307.8146 & 60.944 & 5.8 & 307.8143 & 60.9446 & 307.8164 & 60.9438 & $\mathrm{~N} / \mathrm{A}$ & N/A \\
\hline 145675 & $5 / 7 / 13$ & $4: 29: 02$ & 320.536 & 77.072 & 4 & 320.5401 & 77.0751 & 5.8 & 320.5392 & 77.0739 & 320.5420 & 77.0735 & 320.54 & $77.0747^{10,14}$ \\
\hline 115214 & $5 / 4 / 22$ & $7: 54: 29$ & & & & 324.4306 & 55.7844 & 5.8 & N/A & N/A & N/A & N/A & N/A & N/A \\
\hline 115135 & $5 / 4 / 21$ & $4: 11: 52$ & 307.241 & 73.665 & 4 & 307.2530 & 73.6528 & 5.8 & 307.2591 & 73.6535 & 307.2582 & 73.6529 & 307.2539 & $73.6553^{2}$ \\
\hline$\underline{113120}$ & $5 / 4 / 01$ & 14:20:15 & 247.88 & 2.184 & 4 & 247.8688 & 2.1871 & 5.8 & 247.8696 & 2.1877 & 247.8693 & 2.1869 & 247.87 & $2.1873^{15,20}$ \\
\hline 111622 & $5 / 3 / 19$ & $9: 31: 18$ & 154.157 & 43.566 & 4 & 154.2016 & 43.5463 & 5.8 & 154.1995 & 43.5475 & 154.2004 & 43.5477 & 154.1996 & $43.5485^{19,2}$ \\
\hline 106415 & $5 / 2 / 19$ & 12:40:01 & 166.429 & -40.68 & 4 & 166.4140 & -40.6828 & & 166.4130 & -40.6837 & 166.4127 & -40.6837 & N/A & N/A \\
\hline
\end{tabular}

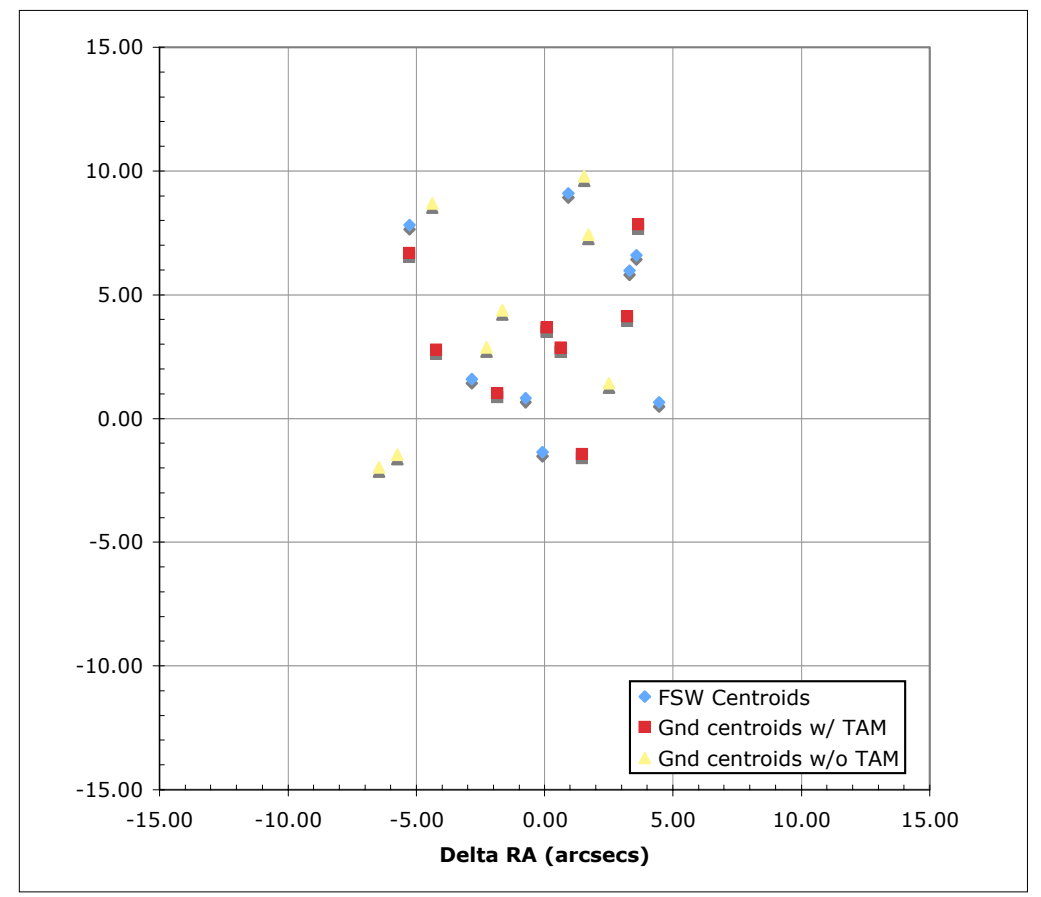

Figure 3.4 Differences in the RA and DEC in arcseconds between the XRT TDRSS position and the ground position of the optical counterpart

Table 3.2 shows the GRBs for which XRT obtained a prompt position. There is no BAT position recorded for trigger 115214 due to a drop out in the TDRSS telemetry. In addition, the XRT telemetered a false centroid position on a cosmic-ray interaction which occurred close to a background X-ray due to the source being too faint for a detection, even in a 2.5 second integration frame.

For the Gamma-ray bursts where the XRT obtained a prompt position and there was a successful optical counterpart identified, we compared the FSW centroid with the optical position. In addition, the Postage Stamp images were reprocessed on the ground with "ximage" to obtain the GRB centroid positions in detector co-ordinates to calculate the

${ }^{5}$ ximage is part of the HEADAS software distributed for XRT data analysis http://swift.gsfc.nasa.gov/docs/swift/analysis 
RA and DEC with and without the TAM correction. This allows the comparison of the best possible position that can be obtained from the data with the position calculated onboard. The results are shown in Figure 3.4. The plot shows that the flight software produces positions with comparable accuracy to those obtained from the images on the ground and therefore we believe the accuracy is at the statistical limit for a given source brightness. The plot shows the differences between the positions have virtually zero offset in RA and a few arcsec offset in Dec, within the statistics of a few bursts. These positions will be adjusted for the updated boresight offset and re-compared with the optical counterpart positions. The centroids obtained from the ground software without the TAM correction show slightly more dispersion than the flight software centroids and the ground centroids obtained with the TAM correction. The two positions with an offset of $\sim 9$ arcsecs were obtained when the spacecraft had not quite settled sufficiently at the time the exposures were taken, and therefore the images are smeared reducing the position accuracy. Positions with increased accuracy are obtained from data taken later in Photon-Counting Mode, where there are more counts and the spacecraft has completely stabilised, although currently the TAM correction is not available in the 'xrtpipeline' ${ }^{6}$ for these data.

\subsection{XRT Observations of GRB 050714b}

The observations of GRB $050414 \mathrm{~b}$ are a good example of the unique observing capabilities of the XRT. In this section, we describe the modes and the data products obtained during the early observations of this burst; during these observations the photodiode modes were de-activated ${ }^{7}$ and therefore no LrPD or PuPD data were obtained.

\subsubsection{XRT Position}

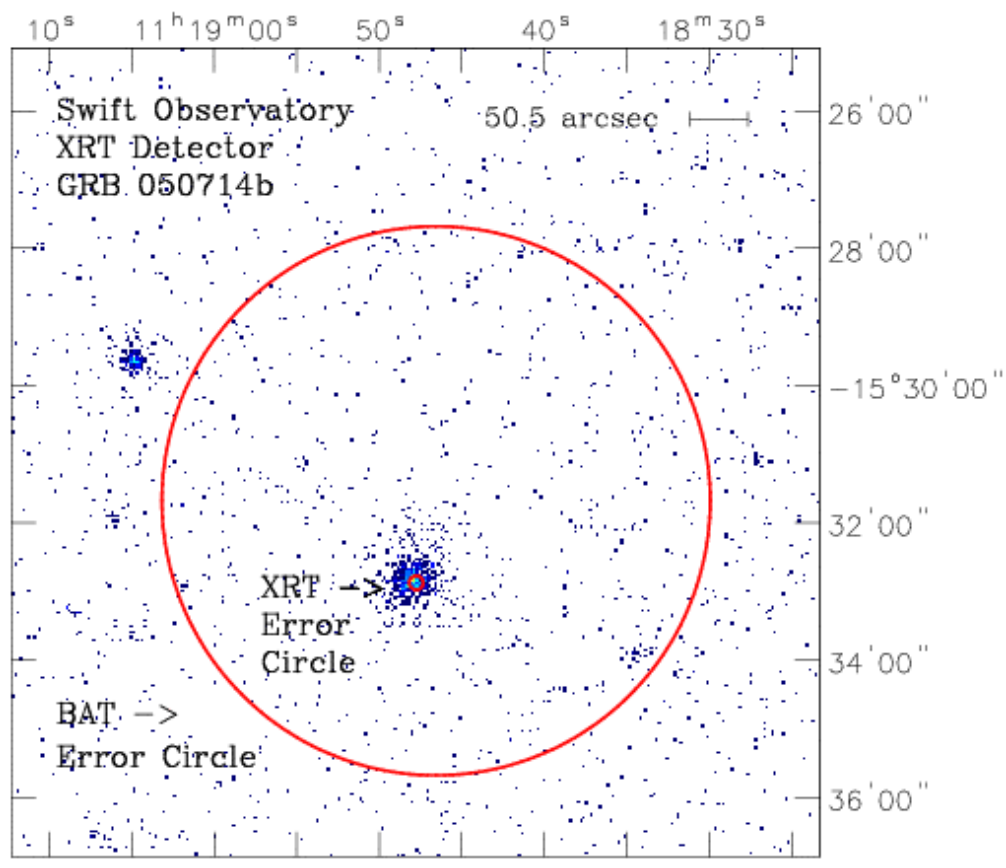

Figure 3.5 Photon-Counting image of GRB 050714b (bottom right) from 22 ksecs of observation over multiple orbits, showing a serendipitous source in the upper left of the figure.

The BAT triggered on the burst at $22: 40: 32$ on the $14^{\text {th }}$ July 2005 . The observatory immediately slewed to point the narrow field instruments at the GRB and settled around 150 seconds later. The XRT immediately switched into Image Mode to obtain a position. The source was not bright enough to obtain a centroid from a 0.1 second exposure, but 94 pixels were above the event threshold in the 2.5 second image and a successful position was obtained. The BAT and

${ }^{6}$ ximage is part of the HEADAS software (http://swift.gsfc.nasa.gov/docs/swift/analysis)

${ }^{7}$ Following damage to the CCD due to a probable micro-metroid event on $27^{\text {th }}$ May 2005, the photodiode modes were de-activated by setting the event threshold to 4094. The modes will be re-enabled following the calibration of the amount of charge injection from new hot pixels with changing temperature ${ }^{16}$. 
XRT error circles are shown, superimposed on the 22 ksecond PC Mode data in Figure 3.5. The XRT onboard position is well within the BAT error circle and is less than an arcsec from the optical position.

\subsubsection{XRT Light-curves}

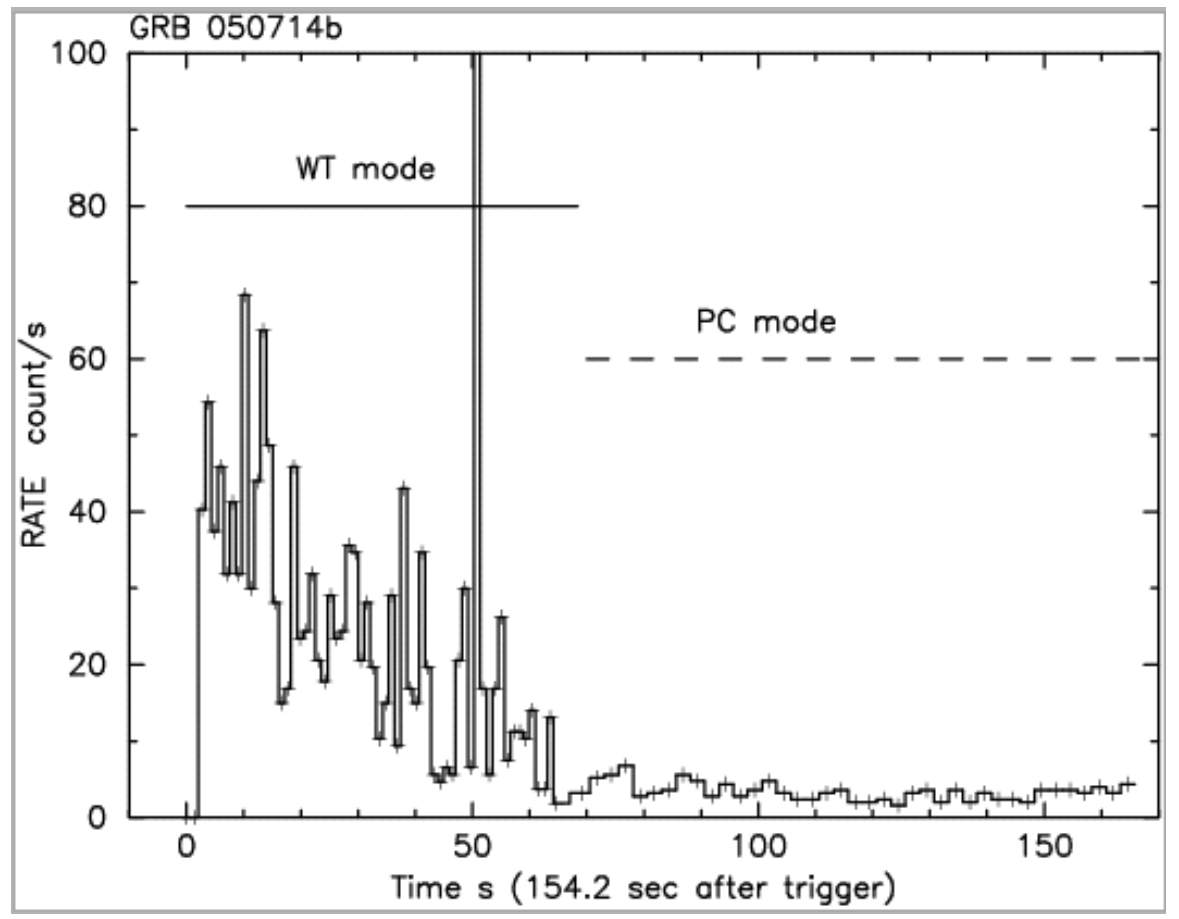

Figure 3.6 Raw unprocessed TDRSS light-curve for GRB 050714b

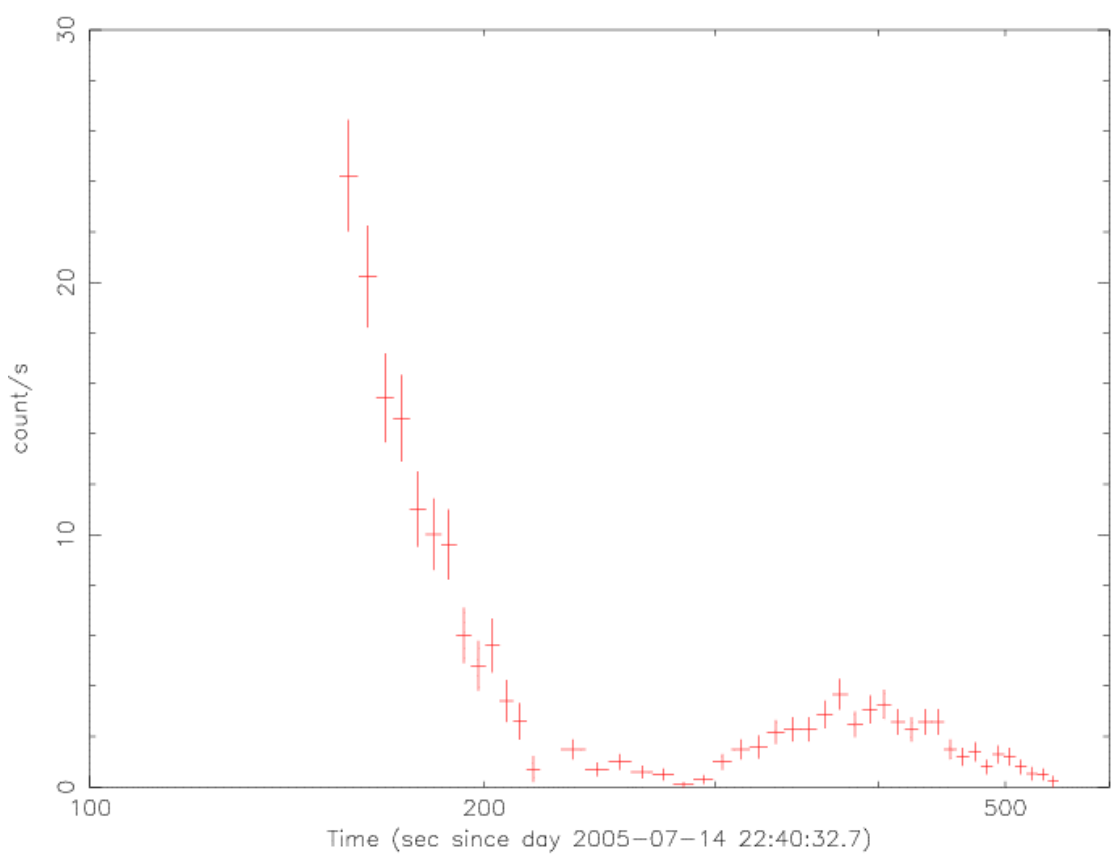

Figure 3.7 Processed light-curve for GRB050714b, WT Mode and PC Mode, all grades

Following Image Mode, the XRT switched through PuPD and LrPD Mode and into Window Timing Mode. When the count-rate dropped sufficiently, the XRT switched into PC Mode. During this time a TDRSS light-curve was obtained; 
the first two bins are zero due to the de-activation of the photodiode modes, but the remainder of the unprocessed TDRSS light-curve can be seen in Figure 3.6.

Processing the data from the solid-state recorder and reconstructing the events produces a continuous light-curve from WT to PC Mode; this is shown in Figure 3.7. Due to the uncontrolled on-orbit temperature, the flight software countrate switch point for PC Mode to WT Mode has been increased from 5 to 10 counts above threshold to prevent mode 'flip-flopping' at high temperatures. Mode flip-flopping occurs due to hot pixels in PC Mode increasing the count-rate so that the FSW switches to WT Mode. Due to the fast clocking in WT Mode, the charge due to the hot pixels is below the event threshold and therefore the count-rate is sufficiently low to switch back into PC Mode. There are a few seconds of dead time during the mode switching and therefore switching back and forth between modes is undesirable. An additional count-rate window and count-rate threshold have been added to PC Mode to try and minimize the effect of the hot pixels at increased temperatures. These parameters are currently being optimized and therefore the switch point from PC to WT Mode has not yet been reduced. For this reason, during the flare at 300 seconds the XRT remained in PC Mode and at a count-rate of greater than 1 count/second the data are piled-up. From the analysis of the wings of the Point Spread Function (PSF) it is possible to account for the pile-up and recover the full spectroscopic accuracy; the data shown here are not corrected for the affects of pile-up.

\subsection{XRT Spectra}

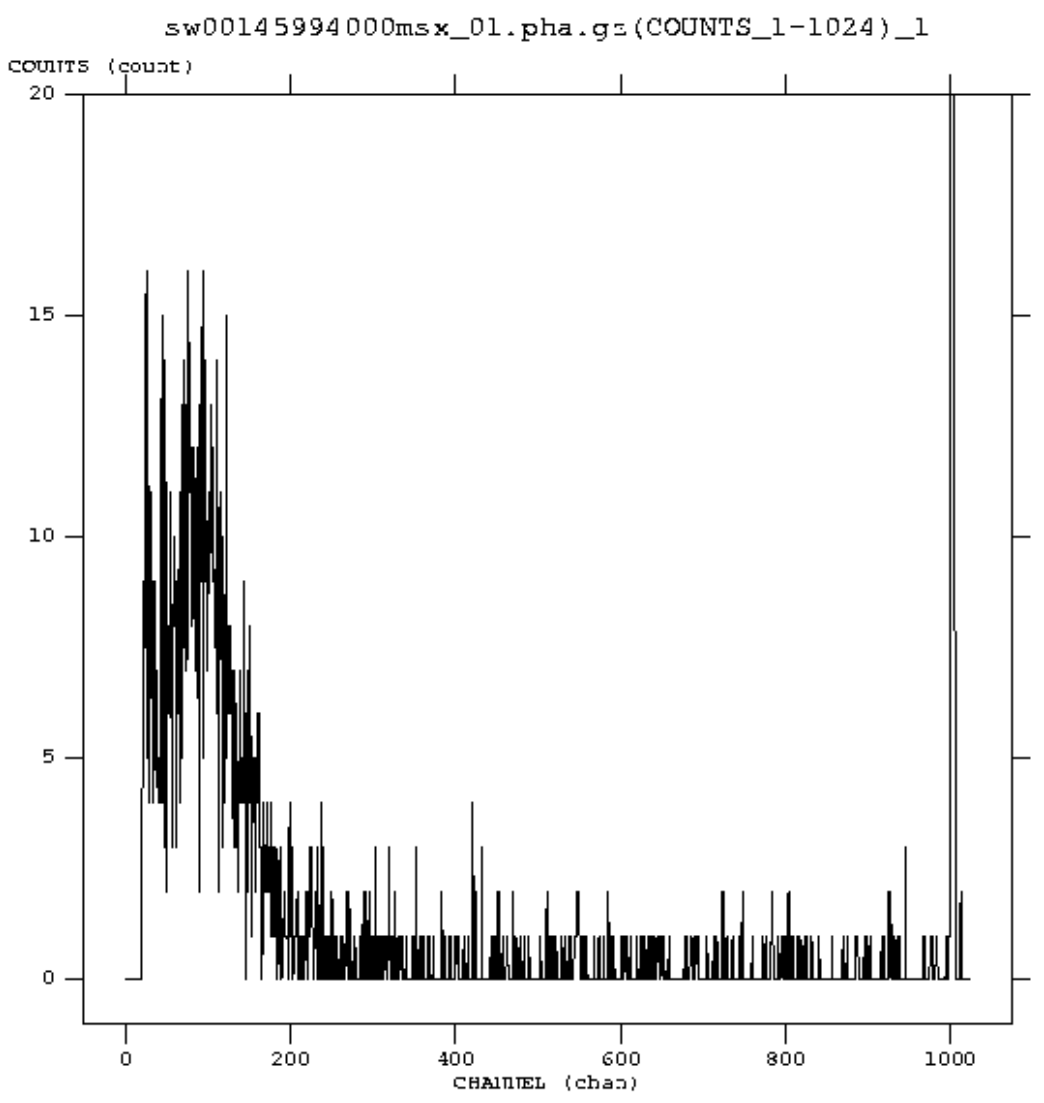

Figure 3.8 Raw unprocessed TDRSS Windowed Timing spectrum 


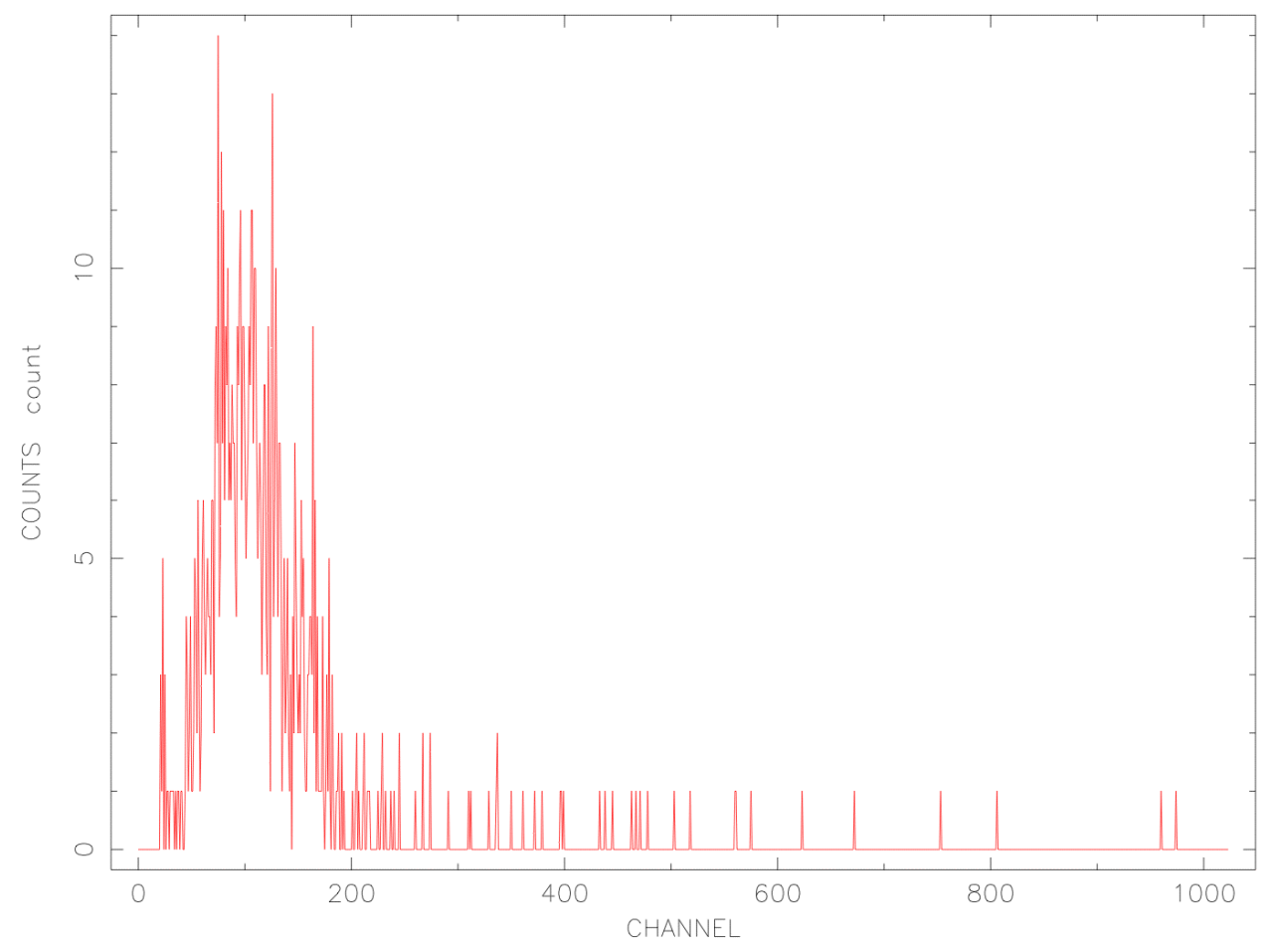

Figure 3.9 Windowed Timing spectrum for GRB 050714b, all grades

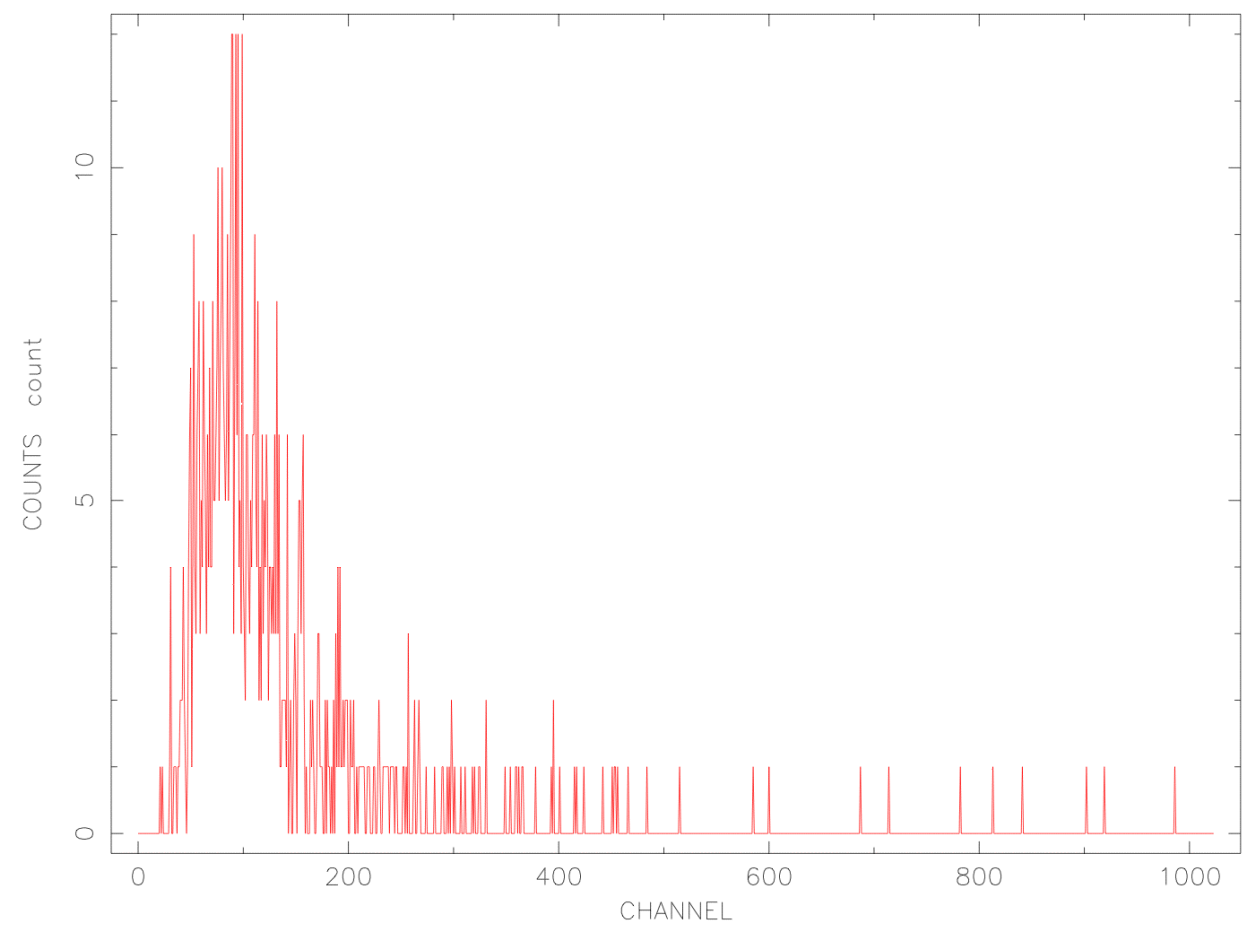

Figure 3.10 Photon-Counting Spectrum for GRB050714b, all grades 
The XRT produces two TDRSS spectra for each burst, one from LrPD Mode and one from WT Mode. Because of the configuration of LrPD Mode at that time, only the WT Mode TDRSS spectrum contains any events; this is shown in Figure 3.8. The data are not reconstructed into events onboard, so the TDRSS spectrum is really a histogram of all the pixels above the event threshold of $80 \mathrm{dn}$. The 4095 ADC channels are binned by a factor of four before being telemetered. The WT Mode spectrum obtained from processing the solid-state recorder data can be seen in Figure 3.9. To allow a comparison with the TDRSS spectrum, the processed spectrum contains all event grades. In addition, the PC Mode data from the 22 ksecond observation is shown in Figure 3.10. This is also for all grades. It can be seen from all three spectra that the majority of the energy from the GRB is below $2 \mathrm{keV}$. The lower energy threshold is $200 \mathrm{eV}$ in order to limit the effects of the bright Earth limb as the observations approach the Earth constraint.

\section{SUMMARY AND FURTHER WORK}

The XRT is achieving all of its science goals and revealing some unexpected features of GRBs. The loss of the XRT controlled cooling 10 days after launch created some operational difficulties, but through the optimisation of the science parameters available in the flight software at launch, in conjunction with the Build 8.9 software uploaded on the $26^{\text {th }}$ May 2005, the XRT has continued to meet all of its science goals at temperatures as high as $-44^{\circ} \mathrm{C}$. Damage to the CCD on $27^{\text {th }}$ May 2005 has added some additional complications to the operation of Photodiode Mode, but the continuous bias update and the temperature dependent threshold table will allow the detrimental effects to be calibrated out. Further optimisations of the hot pixel table, and the count-rate threshold and count-rate window in PC Mode will be made, to remove undesirable mode switching.

\section{ACKNOWLEDGEMENTS}

This work is supported at Penn State by NASA contract NAS5-00136; at the University of Leicester by the Particle Physics and Astronomy Research Council on grant number PPA/Z/S/2003/00507; and at OAB by funding from ASI on grant number $\mathrm{I} / \mathrm{R} / 039 / 04$. We gratefully acknowledge the contributions of dozens of members of the Swift team at PSU, University of Leicester, OAB, GSFC, ASDC, SwRI and Swales who helped make this Observatory possible and to the Flight Operations Team for their dedication and support.

\section{REFERENCES}

1. Barthelmy, S. et al., "The Burst Alert Telescope (BAT) on the Swift MIDEX Mission", 2005, Space Science Review, 120, in press

2. Bloom, J.S., GRB 050421: IR Observations, GCN 3306

3. Burrows, D.N. et al., "The Swift X-ray Telescope", 2005, Space Science Review, 120, in press

4. Burrows, D.N. et al., "Swift XRT and VLT Observations of GRB 041223", 2005, Astrophysical Journal, 622, L85

5. Covino, S., D’Avanzo, P., Malesani, D., Israel, G.L., Piranomonte, S., Tagliaferri, G., Chincarini, G. and Stella, L., "GRB 050714b: candidate afterglow does not fade", GCN 3616

6. Covino, S., D’Avanzo, P., Bagnulo, S., Szeifert, T., Malesani, D., Tagliaferri, G., Chincarini, G., Piranomonte, S., Antonelli, L.A. and Stella, L., "GRB 050721a: Optical afterglow confirmed", GCN 3657

7. Cusumano, G. et al., "Absolute timing with the Swift X-ray Telescope", 2005, Proc. SPIE, 5898, these proceedings

8. Gehrels, N. et al., "The Swift Gamma-ray Burst Mission", 2004, The Astrophysical Journal, 611, 1005

9. Goad, M., et al., "Swift observations of the prompt X-ray emission and afterglow from GRB 050126 and GRB 050219a", 2005, The Astrophysical Journal, submitted

10. Hearty, F., Stringfellow, G., Lamb, D.Q., York, G., Wallerstein, G., Woolf, V., Anderson, S., Dembicky, J., Barentine, J., McMillan, R., Ketzeback, B. and Rechart, D., "GRB 050713: ARC NIR Detections and Identification of Fading”, GCN 3583

11. Hill, J.E. et al., "Readout modes and automated operation of the Swift X-ray Telescope", 2004, Proc. SPIE, 5165, 217

12. Hill, J.E. et al., "GRB 050117: Simultaneous Gamma-ray and X-ray observations with the Swift satellite", 2005, The Astrophysical Journal, submitted

13. Kennea, J.A. et al., "Controlling the Swift XRT CCD temperature via passive cooling", 2005, Proc. SPIE, 5898, these proceedings

14. Malesani, D., D’Avanzo, P., Palazzi, E., Israel, G.L., Chincarini, G., Stella, L. and Pedani, M., "GRB 050713: possible I-band afterglow", GCN 3582

15. McNaught, R. and Price, P.A., "GRB 050401: Optical afterglow candidate”, GCN 3163 
16. Morris, D.C. et al., "Temperature dependent calibration products of the Swift X-ray Telescope", 2005, Proc. SPIE, 5898 , these proceedings

17. Osborne, J.P. et al., "The in-flight spectroscopic performance of the Swift X-ray Telescope", 2005, Proc. SPIE, 5898 , these proceedings

18. Roming, P. et al., 2005, “The Swift Ultra-Violet/Optical Telescope," Space Science Review, 120, in press

19. Rykoff, E., Shaefer, B. and Quimby, R., "GRB 050319: Detection of Possible Optical Counterpart”, GCN 3116

20. Rykoff, E.S., Yost, S.A. and Smith, D.A., "GRB 050401: ROTSE-III Detection of Prompt Optical Counterpart", GCN 3165

21. Rykoff, E.S., Yost, S.A. and Swan, H., "GRB 050525a: ROTSE-III Detection of possible counterpart”, GCN 3465

22. Rykoff, E.S., Yost, S.A., Swan, H. and Quimby, R., "GRB 050525a: ROTSE-III Refined Analysis", GCN 3468

23. Tanvir, N. et al., "GRB 050716: UKIRT identification of a candidate afterglow", GCN 3632

24. Tagliaferri, G. et al., "An unexpectedly rapid decline in the X-ray afterglow emission of long gamma-ray bursts, 2005, Nature, in press

25. Yoshioka, T., Chen, C.W., Nishiura, S., Isogai, M., Soyano, T., Chen, W.P., Urata, Y., Tamagawa, T., Huang, K.Y., Ip, W.H., Qiu, Y., and Lou, Y.Q., "GRB 050319: Confirmation of optical afterglow”, GCN3120 